\title{
Intestinal hyperpermeability: a gateway to multi-organ failure?
}

\author{
QiQ̨i Zhou ${ }^{1,2}$ and G. Nicholas Verne ${ }^{1}$
}

'Department of Medicine, Tulane University School of Medicine, New Orleans, Louisiana, USA. ${ }^{2}$ Malcom Randall VA Medical Center, Research Service, Gainesville, Florida, USA.

\begin{abstract}
In critically ill patients, disruption of intestinal epithelial cell function occurs due to exposure of the epithelium to toxic internal and external inflammatory stimuli, which are key factors that trigger sepsis and multiorgan dysfunction syndrome (MODS). A greater understanding of how trauma and gut failure lead to sepsis and progression to MODS is much needed. In this issue of the $J C l$, Armacki and colleagues identify mechanisms by which thirty-eight-negative kinase 1 (TNK1) promotes the progression from intestinal apoptosis and gut failure to bacterial translocation, sepsis, and MODS. Moreover, the results of this study suggest TNK1 as a potential therapeutic target to prevent sepsis and MODS.
\end{abstract}

\section{Intestinal hyperpermeability and disease states}

In health, the major function of the epithelial lining of the gastrointestinal tract is to act as a filter, allowing absorption of required nutrients, but barring bacteria, macromolecules, and toxic compounds, into the body $(1,2)$. Disruption of this epithelial barrier can lead to local gastrointestinal dysfunction and systemic abnormalities, such as bacterial translocation and sepsis (3). Disturbances in this barrier may also lead to enhanced uptake of a host of toxic substances, including inflammatory molecules, pathogenic bacteria, and antigens, from the intestinal lumen into the bloodstream, thereby promoting a state of chronic low-level inflammation (4). The resulting increase in intestinal permeability (hyperpermeability or leaky gut) allows further translocation of bacteria, antigens, and toxic substances through the mucosal layer of the gut, leading to activation of mucosal immune responses and, subsequently, abdominal pain and diarrhea (5). These inflammatory mediators signal epithelial, neuronal, and muscle cells, leading to intestinal dysfunction (6). It is well established that inflammatory conditions, such as inflammatory bowel disease (IBD), celiac sprue, and acute alcoholic gastroenteritis, are associated with increased gut permeability (7). For these conditions, acute symptoms usually coincide with acute inflammation, which leads to chronic abdominal pain, diarrhea, and bloating. Transient inflammation of the gut and intestinal hyperpermeability may cause a sensitization of the enteric nervous system that persists long after resolution of the inflammation (8-10). These local intestinal inflammatory mediators can also lead to increased intestinal permeability in a subset of patients with postinfectious, diarrhea-predominant irritable bowel syndrome (IBS) (11).

\section{Effect of intestinal hyperpermeability on sepsis and MODS}

Disruption of intestinal epithelial cell function in critically ill patients occurs as the result of exposure of the epithelium to toxic internal and external inflammatory stimuli, which trigger sepsis and multi-organ dysfunction syndrome (MODS). As intestinal permeability increases and proinflammatory cytokines, such as TNF- $\alpha$, IL-1,

Related Article: p. 5056

Conflict of interest: The authors have declared that no conflict of interest exists.

Reference information: / Clin Invest. 2018;128(11):4764-4766. https://doi.org/10.1172/JCI124366.

and IL-6, are released into the systemic circulation, fluid is extravasated from the gut, and extravascular edema occurs (12). Moreover, proinflammatory cytokines induce changes in tight junction proteins in the gut, leading to hyperpermeability (13-15). In addition to the loss of the intestinal barrier function, the microbiome is altered and characterized by an increase in the number of virulent and invasive bacteria, thereby resulting in dysbiosis and a dysregulated immune response that increases systemic inflammation. All of this culminates in enhanced apoptosis, particularly in intestinal and pulmonary epithelial cells. In healthy individuals, the intestine contains a large microbiome populated with commensal bacteria. During sepsis and MODS, this population shifts to include more virulent and pathogenic bacteria, altering the complex crosstalk between the immune system, microbiome, and intestinal epithelium (16). Effectively, the gastrointestinal tract serves to hasten MODS, and altered levels of citrulline and intestinal fatty acidbinding proteins are markedly elevated in critically ill patients. This pathology is further propagated by bacterial translocation from the gut and systemic absorption of endotoxins $(17,18)$. Systemic endotoxins are largely responsible for propagating the inflammatory cascade, and injection of LPS into mice stimulates sepsis and leads to altered epithelial barrier function. Proinflammatory cytokines, including IL-1, IL-6, and TNF- $\alpha$, are subsequently released, leading to endothelial injury and cardiac depression. Figure 1 summarizes the importance of the intestinal barrier and different mechanisms involved in the development of sepsis and MODS. The gut is a major driver of uncontrolled inflammation, proinflammatory cytokine release, and end-organ damage and failure. Intestinal hyperpermeability serves as a bioprotective gateway in the host, via feedback mechanisms, that prevents a deleterious cascade, namely the vicious circle, which exacerbates sepsis and MODS. Important- 


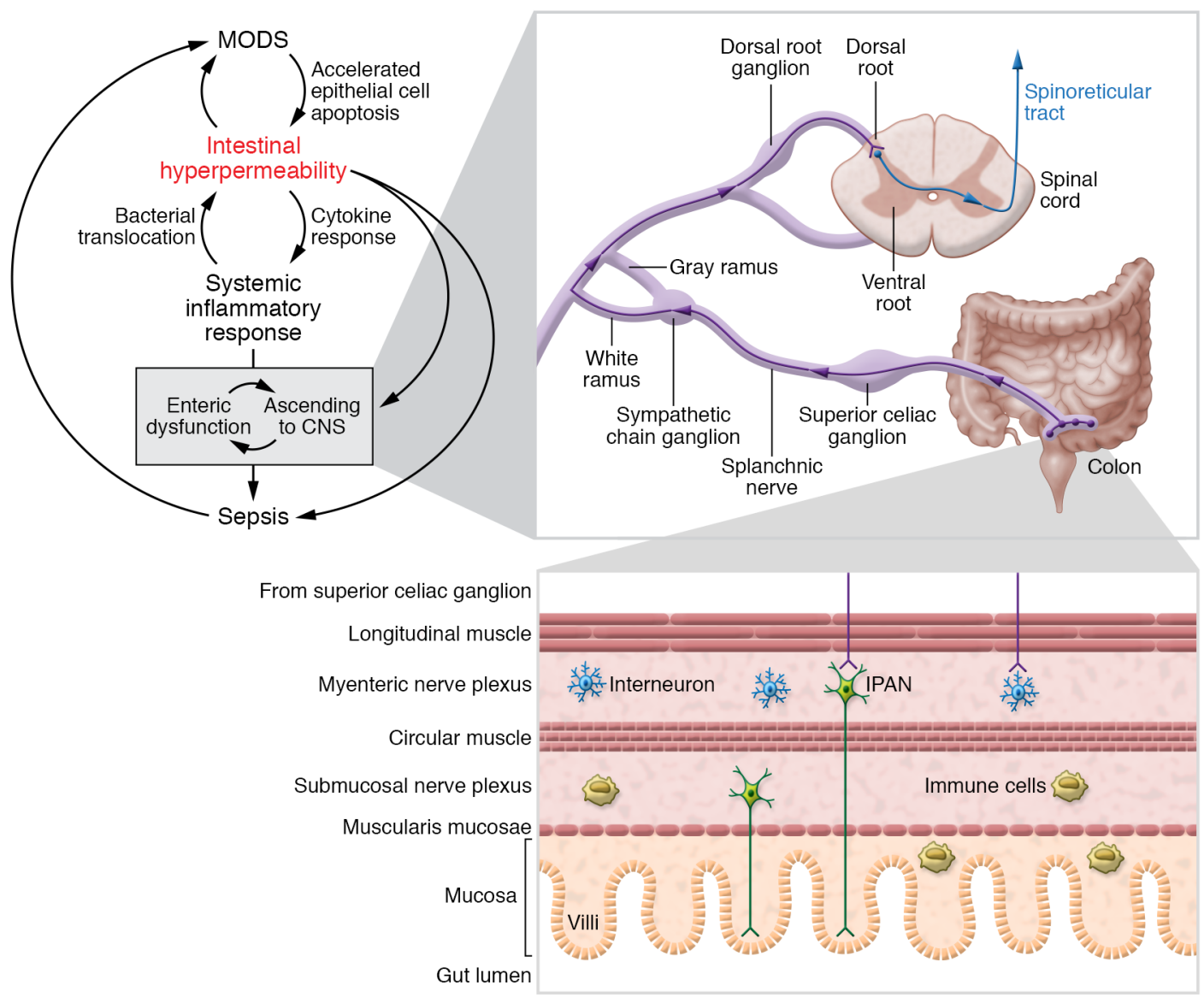

Figure 1. Multi-organ dysfunction syndrome (MODS). Upper left panel: There is a feedback from intestinal hyperpermeability to MODS that causes an acceleration of epithelial cell apoptosis. Intestinal hyperpermeability opens the gateway of biological protection, thereby allowing bacterial translocation, enhancing cytokine production, and ultimately inducing the systemic inflammatory response. The cascade of the MODS will lead to sepsis through opening of the gateway, via intestinal hyperpermeability facilitated by enteric dysfunction and then the transfer of the local injury information to the CNS. The upper right panel shows how the colon links with the CNS, and the lower right panel shows the details of the colon from mucosa to myenteric nerve plexus. IPAN, intrinsic primary afferent neuron.

ly, intestinal hyperpermeability, as an open biogateway, via toxin and cytokine release, causes nociceptive ascending input to the CNS, which may in turn cause neuroinflammation and facilitate MODS.

\section{Trauma-induced intestinal injury and multi-organ failure}

MODS is a significant cause of mortality in patients with sepsis and represents the final common pathway that results from the systemic inflammatory response syndrome in critically ill patients, such as those suffering from burn injuries, trauma, hemorrhagic shock, and pancreatitis (19). Organs that contain major epithelial barriers, such as the gastrointestinal tract, lungs, kidney, and liver, are most commonly affected by sepsis and MODS. Despite the high mortality associated with MODS, the pathogenetic mechanisms that lead to MODS appear to be multifactorial and are not well elucidated. In critically ill patients, intestinal injury and the resulting intestinal hyperpermeability leads to transmural bacterial translocation from the gut to the systemic circulation, and the uptake of systemic inflammatory mediators and cytokines are contributing factors (20). Sepsis is now clearly recognized as a cause of organ failure that results directly from a dysregulated host response to inflammation and infection (21). Even with early recognition of sepsis, patients often go on to develop MODS, which is characterized by inflammatory and systemic damage to critical organs. Moreover, the transition from sepsis to MODS occurs despite early initiation of antimicrobial therapy, volume resuscitation, and circulatory support with vasopressors. MODS also has serious consequences and extremely high mortality and morbidity despite aggressive interventions.
In this issue, Armacki and colleagues (22) propose that thirty-eight-negative kinase 1 (TNK1), a member of the ACK family of kinases that was first isolated from $\mathrm{CD} 4^{+} \mathrm{Lin}^{-} \mathrm{CD} 38^{-}$stem/progenitor cells (23), may be an important mediator of apoptosis and subsequent organ failure. TNK1 transcripts are found in fetal tissues, including the gastrointestinal tract, but only in a few adult tissues, including the small intestine, colon, prostate, ovaries, and testis. There is also some evidence that TNK1 may play a role in differentiation of the gastrointestinal tract, particularly the small and large intestine (24). Armacki et al. studied the effect of TNK1 on intestinal integrity and its potential role in MODS using a Tnk1-knockin mouse model that harbors a tetracycline-inducible active form of TNK1 at the X-chromosomal Hprt locus. In this model, TNK1 
expression induced crypt-specific apoptosis, leading to bacterial translocation, septic shock, and early death. In vivo, TNK1 led to STAT3 phosphorylation, nuclear translocation of p65, and subsequent release of IL- 6 and TNF- $\alpha$. Perhaps even more interestingly, gut-specific deletion of Tnk1 protected intestinal mucosa from experimental colitis induced by $4 \%$ dextran sodium sulphate (DSS) and prevented cytokine release in the gut. Moreover, TNK1 was also deregulated in the gut of murine and porcine trauma models and in patients with IBD. Together, these findings by Armacki et al. are provocative and suggest that TNK1 might be a missing link in the progression from intestinal apoptosis and gut failure to bacterial translocation, sepsis, and MODS. Despite these preliminary findings, caution needs to be taken in extrapolating these results to humans. Future clinical studies that target TNK1 in the intensive care unit (ICU) setting of sepsis and subsequent monitoring are required to determine if this approach is able to prevent multiple organ damage in critically ill patients.

\section{Conclusions and potential strategies to manage MODS and sepsis}

A greater understanding of how trauma and gut failure lead to sepsis and progression of MODS is much needed. The study by Armacki et al. supports a role for TNK1 in the progression from intestinal apoptosis and gut failure to bacterial translocation, sepsis, and MODS and adds another possible mechanism for progression. A number of approaches have been described to prevent sepsis and MODS in critically ill patients, none of which have led to successful therapies. Armacki et al. provide a hypothesis for a potential role for TNK1 as a therapeutic target to prevent sepsis and MODS. Novel therapeutic approaches, such as targeting TNK1, are needed to prevent and/or reverse the del- eterious effects of critical illness in the gut and its consequences. These findings by the authors will need further validation in clinical trials that target TNK1 in the ICU setting of sepsis to prevent multiple organ damage in critically ill patients.

\section{Acknowledgments}

Supported by the National Institute of Diabetes and Digestive and Kidney Diseases (DK099052) and the Department of Veterans Affairs (CX001477-01).

Address correspondence to: G. Nicholas Verne, Tulane University School of Medicine, 1430 Tulane Ave., SL-8512, New Orleans, Louisiana 70112, USA. Phone: 504.988.7800; Email:gverne@tulane.edu.

1. Spiller RC, et al. Increased rectal mucosal enteroendocrine cells, T lymphocytes, and increased gut permeability following acute Campylobacter enteritis and in post-dysenteric irritable bowel syndrome. Gut. 2000;47(6):804-811.

2. Camilleri M, Madsen K, Spiller R, Greenwood-Van Meerveld B, Van Meerveld BG, Verne GN. Intestinal barrier function in health and gastrointestinal disease. Neurogastroenterol Motil. 2012;24(6):503-512.

3. Assimakopoulos SF, et al. Gut-origin sepsis in the critically ill patient: pathophysiology and treatment [published online ahead of print July 12, 2018]. Infection. https://doi.org/10.1007/ s15010-018-1178-5.

4. McClave SA, Lowen CC, Martindale RG. The 2016 ESPEN Arvid Wretlind lecture: The gut in stress. Clin Nutr. 2018;37(1):19-36.

5. Farhadi A, Fields JZ, Keshavarzian A. Mucosal mast cells are pivotal elements in inflammatory bowel disease that connect the dots: stress, intestinal hyperpermeability and inflammation. World J Gastroenterol. 2007;13(22):3027-3030.

6. Macdonald TT, Monteleone G. Immunity, inflammation, and allergy in the gut. Science. 2005;307(5717):1920-1925

7. Merlin D, et al. Colonic epithelial hPepT1 expression occurs in inflammatory bowel disease: transport of bacterial peptides influences expression of MHC class 1 molecules. Gastroenterology. 2001;120(7):1666-1679.

8. Srinivasan S, Wiley JW. New insights into neural injury, repair, and adaptation in visceral afferents and the enteric nervous system. Curr Opin
Gastroenterol. 2000;16(1):78-82.

9. Zhou Q, Souba WW, Croce CM, Verne GN. MicroRNA-29a regulates intestinal membrane permeability in patients with irritable bowel syndrome. Gut. 2010;59(6):775-784.

10. Zhou Q, et al. Decreased miR-199 augments visceral pain in patients with IBS through translational upregulation of TRPV1. Gut 2016;65(5):797-805.

11. Spiller R, Garsed K. Postinfectious irritable bowel syndrome. Gastroenterology. 2009;136(6):1979-1988.

12. Angus DC, van der Poll T. Severe sepsis and septic shock. N Engl J Med. 2013;369(9):840-851.

13. Ma TY, et al. TNF- $\alpha$-induced increase in intestinal epithelial tight junction permeability requires NF- $\mathrm{\kappa B}$ activation. Am J Physiol Gastrointest Liver Physiol. 2004;286(3):G367-G376.

14. Zhou Q, et al. MicroRNA 29 targets nuclear factor- $\kappa \mathrm{B}$-repressing factor and Claudin 1 to increase intestinal permeability. Gastroenterology. 2015;148(1):158-169.e8.

15. Shea-Donohue T, Fasano A, Smith A, Zhao A. Enteric pathogens and gut function: Role of cytokines and STATs. Gut Microbes. 2010;1(5):316-324.

16. Cohen J, et al. Sepsis: a roadmap for future research. Lancet Infect Dis. 2015;15(5):581-614.

17. Klingensmith NJ, Coopersmith CM. The gut as the motor of multiple organ dysfunction in critical illness. Crit Care Clin. 2016;32(2):203-212.

18. Coopersmith CM, et al. Inhibition of intestinal epithelial apoptosis and survival in a murine model of pneumonia-induced sepsis. JAMA. 2002;287(13):1716-1721.

19. Singer M, et al. The third international consensus definitions for sepsis and septic shock (Sepsis-3). JAMA. 2016;315(8):801-810.

20. Haak BW, Wiersinga WJ. The role of the gut microbiota in sepsis. Lancet Gastroenterol Hepatol. 2017;2(2):135-143.

21. Sauaia A, Moore FA, Moore EE. Postinjury inflammation and organ dysfunction. Crit Care Clin. 2017;33(1):167-191.

22. Armacki M, et al. Thirty-eight-negative kinase 1 mediates trauma-induced intestinal injury and multi-organ failure. J Clin Invest. 2018;128(11):5056-5072.

23. Hoehn GT, et al. Tnk1: a novel intracellular tyrosine kinase gene isolated from human umbilical cord blood CD34 ${ }^{+} / \mathrm{Lin}^{-} / \mathrm{CD} 38^{-}$stem/progenitor cells. Oncogene. 1996;12(4):903-913.

24. Azoitei N, Brey A, Busch T, Fulda S, Adler G, Seufferlein T. Thirty-eight-negative kinase 1 (TNK1) facilitates TNFalpha-induced apoptosis by blocking NF- $\mathrm{BB}$ activation. Oncogene. 2007;26(45):6536-6545. 